\title{
The recurrent distal 22q11.2 microdeletions are often de novo and do not represent a single clinical entity: a proposed categorization system
}

\author{
Fady M. Mikhail, MD, PhD', Rachel D. Burnside, PhD², Brooke Rush, MS², Jennifer Ibrahim, MD², \\ Robin Godshalk, MS³ ${ }^{3}$ S. Lane Rutledge, MD1, Nathaniel H. Robin, MD1, Maria D. Descartes, MD ${ }^{1}$ and \\ Andrew J. Carroll, PhD ${ }^{1}$
}

Purpose: The five segmental duplications (LCR22-D to -H) at the distal region of chromosome 22 band q11.2 in the region immediately distal to the DiGeorge/velocardiofacial syndrome deleted region have been implicated in the recurrent distal 22q11.2 microdeletions. To date, the distal 22q11.2 microdeletions have been grouped together as a single clinical entity despite the fact that these deletions are variable in size and position depending on the mediating LCR22s.

Methods: Here, we report 13 new unrelated patients with variable size deletions in the distal 22q11.2 region as shown by cytogenomic array analyses. We compare our patients' clinical features with those of previously reported cases to better dissect the phenotypic correlations based on the deletion size and position.

Results: Six patients had the 1.1-Mb deletion flanked by LCR22-D and $-\mathrm{E}$, and presented clinically with a phenotype consistent with

\section{INTRODUCTION}

Recurrent microdeletions and microduplications, also termed recurrent copy-number variations (CNVs), have been identified as a common cause of developmental delay (DD), intellectual disability (ID), autism spectrum disorders, other neuropsychiatric phenotypes, and/or multiple congenital anomalies. ${ }^{1-3}$ The great majority of these recurrent CNVs are mediated by recombination between nonallelic homologous segmental duplications, also called low copy repeats (LCRs), through the well-established mechanism of nonallelic homologous recombination. ${ }^{4}$ In recent years, the detection of these recurrent events has been facilitated by the wide use of cytogenomic arrays in clinical diagnostics. Certain regions of the human genome are enriched with multiple LCR clusters that contain several modules with a very high degree of sequence homology ( $>95 \%$ ), which results in genomic instability of these regions. ${ }^{3}$ Examples include 1q21.1, 7q11.23, 8p23.1, 15q11.2q13.1, 15q13.3, 16p13.11, 16p11.2, 17p12, 17p11.2, 17q11.2, 17q21.31, and 22q11.2.

Eight LCR clusters have been identified in the proximal region of $22 \mathrm{q}$ at band q11.2, and have been named LCR22-A to H. The four centromeric LCR22s (LCR22-A to D) are implicated in the recurrent DiGeorge/velocardiofacial syndrome (DG/VCFs) previously reported cases with distal 22q11.2 microdeletions. Three patients had the $1.8-\mathrm{Mb}$ deletion flanked by LCR22-D and -F, and presented with a similar phenotype. Four patients had the $700-\mathrm{kb}$ deletion flanked by LCR22-E and -F, and presented with a milder phenotype that lacked growth restriction and cardiovascular defects.

Conclusion: We suggest that the recurrent distal 22q11.2 microdeletions do not represent a single clinical entity, and propose categorizing these deletions into three types according to their genomic position. All three deletion types are thought to be pathogenic and are most often de novo. They all share some presenting features but also have their unique features and risks.

Genet Med advance online publication 13 June 2013

Key Words: CNVs; distal 22q11.2 microdeletion; LCR22s; rhabdoid tumors; SMARCB1

microdeletion (OMIM 188400 and 192430), and its reciprocal microduplication (OMIM 608363). Based on the mediating LCR22s, about $90 \%$ of patients with the DG/VCFs microdeletion have the common $\sim 3.0 \mathrm{Mb}$ deletion between LCR22-A and $\mathrm{D}$, whereas $\sim 7 \%$ of the patients have a smaller nested $\sim 1.5 \mathrm{Mb}$ deletion between LCR22-A and B. ${ }^{5}$ These microdeletions in the proximal portion of the 22q11.2 region are the most common recurrent, pathogenic microdeletions in humans with a frequency of approximately 1:4,000 to 1:8,000 live births. ${ }^{6}$

Recently, others and we demonstrated that the five telomeric LCR22s, namely LCR22-D to H, at the distal portion of 22q11.2 are causally implicated in the recurrent distal 22q11.2 microdeletion-associated phenotype(s) (OMIM 611867), and their reciprocal microduplications in the region immediately distal to the DG/VCFs typically deleted region (Figure 2). ${ }^{7-18}$ The structure of the LCR22-D, -E, and -F has been studied and was shown to contain the BCRL module in each LCR22, suggesting that the distal 22q11.2 microdeletions/microduplications are mediated by nonallelic homologous recombination. ${ }^{10}$

To date, the distal 22q11.2 microdeletions have been grouped together as a single clinical entity despite the fact that these deletions are variable in size and position depending on the

\footnotetext{
${ }^{1}$ Department of Genetics, University of Alabama at Birmingham, Birmingham, Alabama, USA; ${ }^{2}$ Laboratory Corporation of America, Research Triangle Park, North Carolina, USA; ${ }^{3}$ Department of Pediatrics, Division of Genetics, St. Joseph’s Children’s Hospital, Paterson, New Jersey, USA. Correspondence: Fady M. Mikhail (fmikhail@uab.edu) 


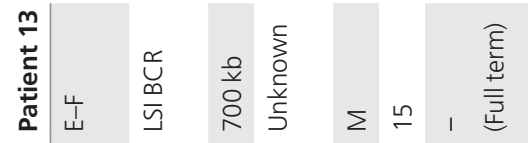

$\simeq$

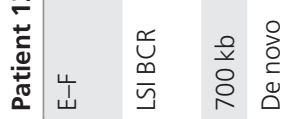

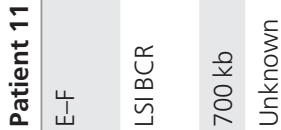

우

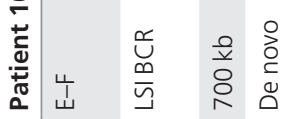

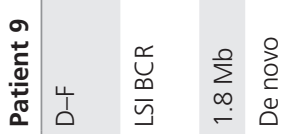

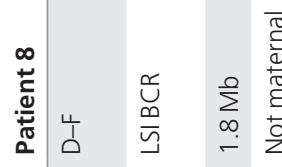

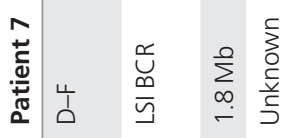

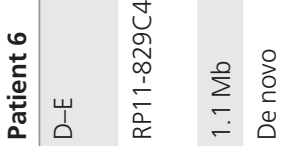

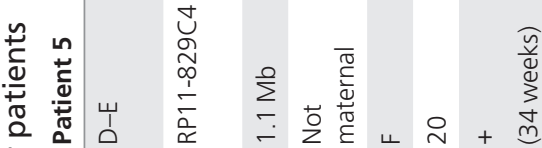

亏े

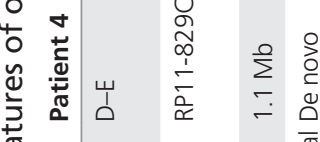

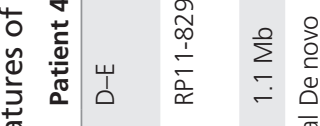

$\stackrel{\pi}{\stackrel{0}{4}} m$

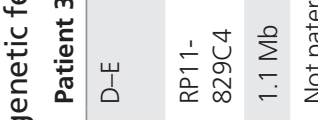

艺

$\frac{7}{\frac{\pi}{2}}$

$\frac{0}{0}$

๕े

高

$\frac{0}{\frac{0}{6}}$

.

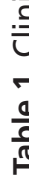

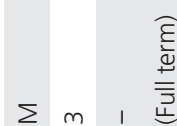

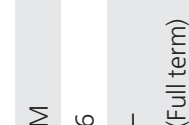

竞

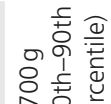

m造范

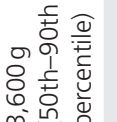

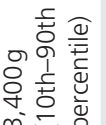

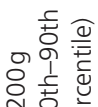

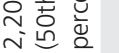

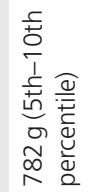

劳

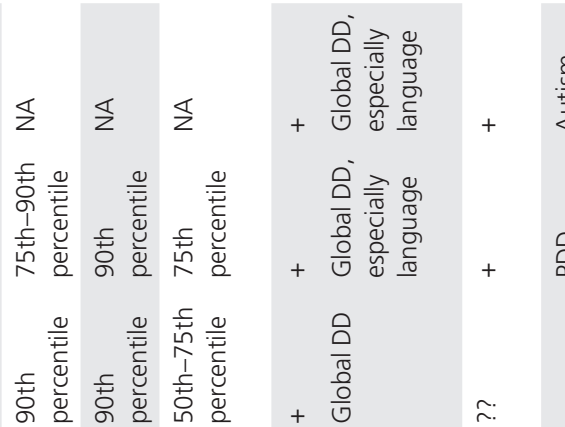

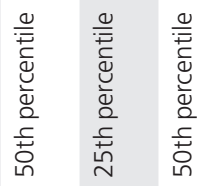

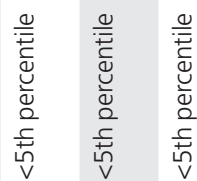

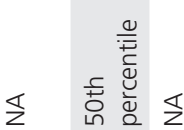

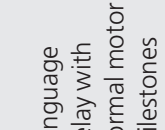

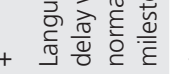

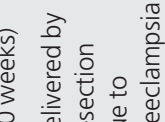

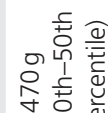

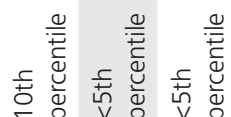

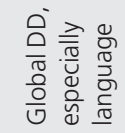

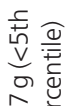

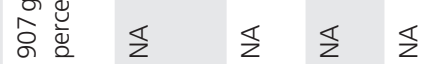

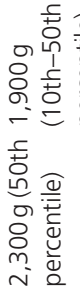

$\stackrel{\pi}{z} \frac{\pi}{2}$

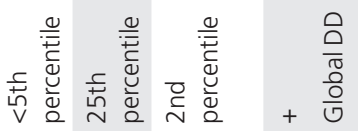

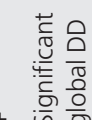

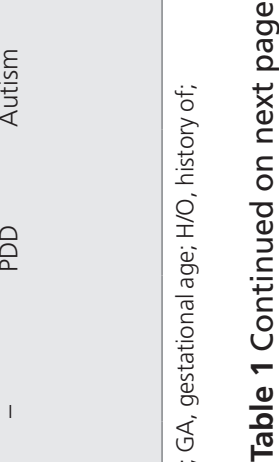

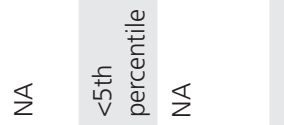




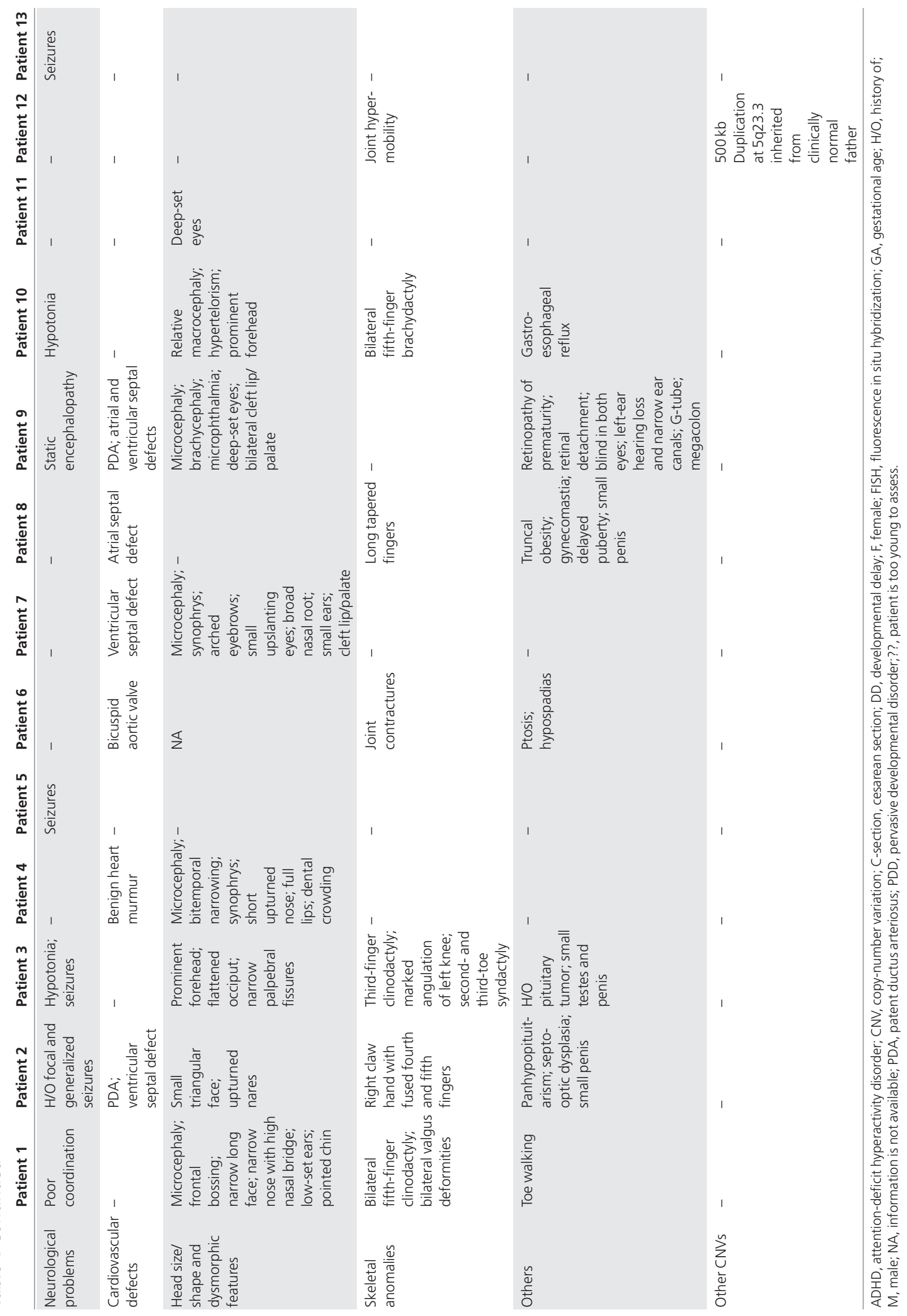


Table 2 Chromosome 22 genomic coordinates of LCR22-A to $-\mathrm{H}$ in human genome builds hg18 and hg19

\begin{tabular}{lcc} 
LCR22 & NCBI36/hg18 & GRCh37/hg19 \\
\hline A & $\sim 17,020,000-17,290,000$ & $\sim 18,640,000-18,910,000$ \\
B & $\sim 18,630,000-19,060,000$ & $\sim 20,250,000-20,680,000$ \\
C & $\sim 19,350,000-19,420,000$ & $\sim 21,020,000-21,090,000$ \\
\hline D & $\sim 19,800,000-20,250,000$ & $\sim 21,470,000-21,920,000$ \\
E & $\sim 21,290,000-21,380,000$ & $\sim 22,960,000-23,050,000$ \\
F & $\sim 21,980,000-22,150,000$ & $\sim 23,650,000-23,820,000$ \\
G & $\sim 22,960,000-23,030,000$ & $\sim 24,630,000-24,700,000$ \\
H & $\sim 23,325,000-23,410,000$ & $\sim 24,995,000-25,080,000$
\end{tabular}

mediating LCR22s, and could be either partially overlapping or nonoverlapping, which suggests that they do not represent a single clinical entity but rather different entities with some difference in their presenting features and risks. In this study, we report 13 new unrelated patients with variable-size deletions in the distal 22q11.2 region as shown by cytogenomic array analyses. We compare our patients' clinical and molecular cytogenetic features with those of previously reported cases in an attempt to better dissect the phenotypic correlations based on the deletion size and position. Finally we propose a system to categorize these deletions based on their genomic position in the distal 22q11.2 region.

\section{MATERIALS AND METHODS}

\section{Patients}

The 13 patients presented in this report were recruited from the clinical cytogenetics laboratories at the University of Alabama at Birmingham (UAB) Department of Genetics and the Laboratory Corporation of America (LabCorp). All patients have undergone clinical cytogenomic array testing for indications that included DD, ID, autism spectrum disorders, dysmorphic features, multiple congenital anomalies, and general suspicion of chromosomal abnormalities. A total of 28 patients were noted to have a deletion in the distal 22q11.2 region in both laboratories among a total of 35,000 patients referred for clinical cytogenomic array testing (3,000 patients at UAB and 32,000 patients at LabCorp), yielding a frequency of $\sim 0.08 \%$. Seventeen patients had the LCR22-D to -E deletion, three patients had the LCR22-D to -F deletion, two patients had the LCR22-D to -G deletion, and six patients had the LCR22-E to -F deletion. Sufficient clinical information was available for the 13 patients reported here. When parents were available, the de novo or inherited origin of the deletion was investigated using fluorescence in situ hybridization (FISH) analyses. This study was approved by the institutional review board for human subject research at UAB.

\section{G-banded chromosome and FISH analyses}

G-banded chromosome and FISH analyses were performed on metaphase preparations of peripheral blood lymphocytes from the patients and their parents using standard techniques. For the
1.1-Mb LCR22-D to -E deletion, the BAC clone RP11-647D11 was used by the cytogenetics laboratory at $\mathrm{UAB}$, whereas the BAC clone RP11-829C4 was used by the cytogenetics laboratory at LabCorp. These clones were prepared from the RPCI11 human genomic library (Empire Genomics, Buffalo, NY), and the clones' identities were confirmed by FISH analyses on normal metaphase spreads as well as end sequencing. For the 1.8-Mb LCR22-D to -F deletion as well as the small $700-\mathrm{kb}$ LCR22-E to -F deletion, the LSI BCR probe (Abbott, Abbott Park, IL) was used by both labs.

\section{Cytogenomic array analyses}

Two cytogenomic array platforms were used. Array comparative genomic hybridization was performed using the $4 \times 44 \mathrm{k}$ or the $8 \times 60 \mathrm{k}$ Agilent oligo-arrays (Agilent Technologies, Santa Clara, CA) by the cytogenetics laboratory at UAB. These are custom-designed arrays that are based on the ISCA (International Standards for Cytogenomic Arrays) consortium design. DNA was extracted from the patients' peripheral blood using the Qiagen Blood Mini Kit (Qiagen, Valencia, CA). DNA labeling, slide hybridization, washing, and scanning were performed following the manufacturer's protocol. The arrays were scanned using the Agilent high-resolution microarray scanner (Agilent Technologies). The scanned arrays were analyzed using the Cytogenomics v1.5 software (Agilent Technologies).

SNP microarray analysis was performed by the cytogenetics laboratory at LabCorp, using either the Affymetrix v6.0 or the Affymetrix Cytoscan HD platform (Affymetrix, Santa Clara, CA). DNA labeling, slide hybridization, washing, and scanning were performed following the manufacturer's protocol. The Affymetrix v6.0 chip contains more than 900,000 SNP probes and 900,000 nonpolymorphic copy-number probes with a median spacing of $0.7 \mathrm{~kb}$, and the data were analyzed using the Affymetrix Genotyping Console Browser v.3.01. The Affymetrix Cytoscan HD contains 743,000 SNP probes and 1,953,000 nonpolymorphic copy-number probes with a median spacing of $0.88 \mathrm{~kb}$, and the data were analyzed using Chromosome Analysis Suite (Affymetrix).

\section{RESULTS}

Thirteen new unrelated cases were found to have deletions in the distal 22q11.2 region by clinical cytogenomic array testing and were confirmed by metaphase FISH analyses. The patients' clinical and molecular cytogenetic features are summarized in Table 1. Table 2 illustrates the genomic coordinates of LCR22-A to $-\mathrm{H}$ in human genome builds hg18 and hg19.

The first significant finding observed is the clear trend for these deletions to be de novo in origin. Parental FISH testing demonstrated that in six patients the deletion was de novo. For three patients, only one parent was available and these were all shown not to have the deletion. In the four remaining patients, the parents were either unavailable or declined parental FISH testing. The second significant observation is the genotypic variability of these deletions. Six patients had the $\sim 1.1 \mathrm{Mb}$ deletion flanked by LCR22-D and -E, three patients had the larger 


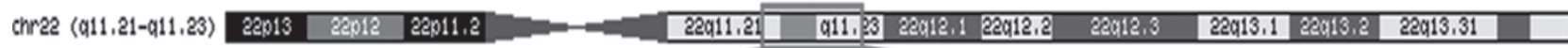

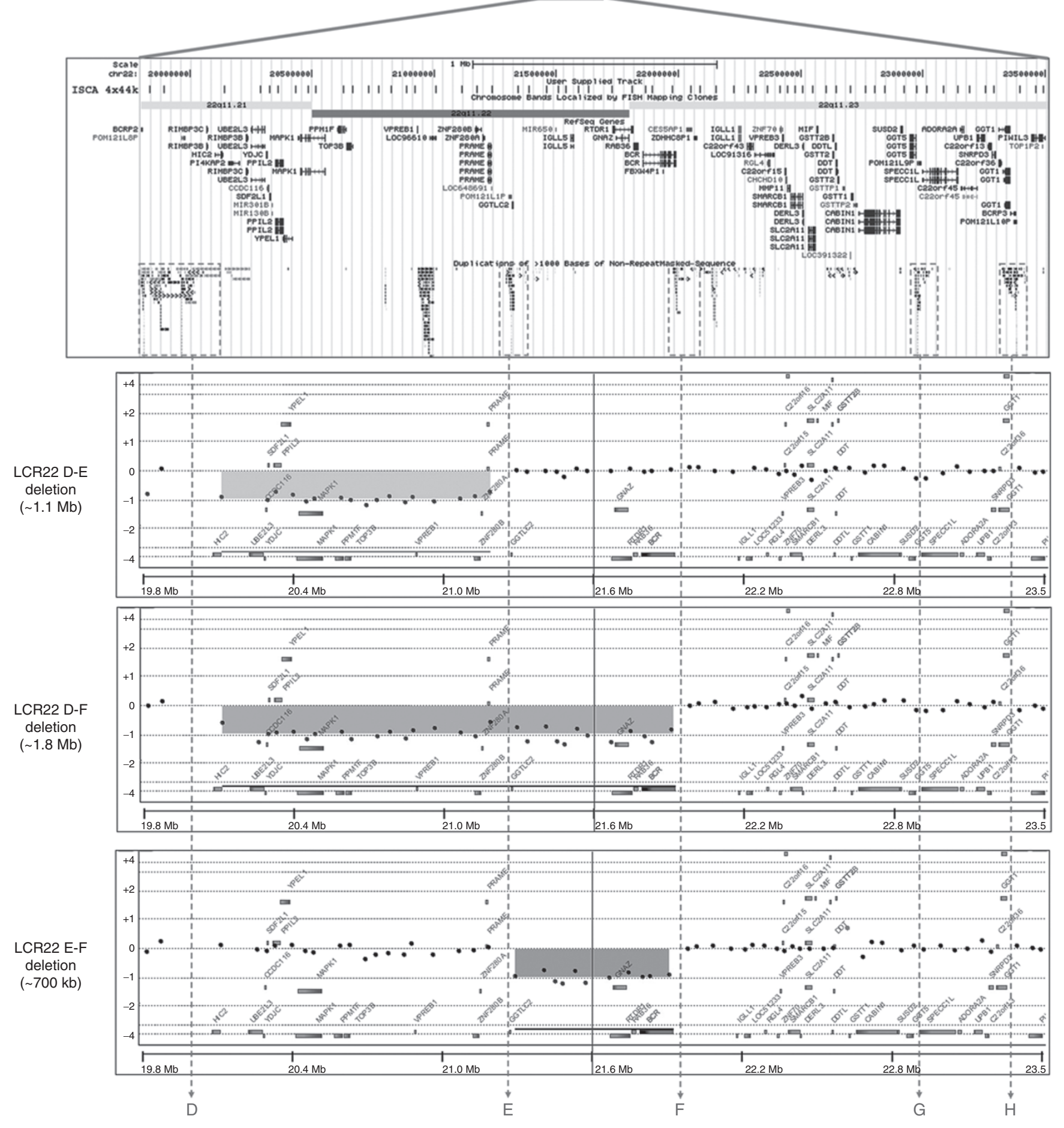

Figure 1 Genomic map of the distal 22q11.2 region (generated using the UCSC genome browser, hg18) aligned with array comparative genomic hybridization plots of overlapping distal 22q11.2 deletions of varying sizes (shaded areas). The dashed boxes and vertical lines represent the LCR22-D to - H.

$\sim 1.8 \mathrm{Mb}$ deletion flanked by LCR22-D and -F, and four patients had the small $\sim 700 \mathrm{~kb}$ deletion flanked by LCR22-E and $-\mathrm{F}$ (Figures 1 and 2). No other clinically significant CNVs were noted in any patient, with the exception of patient 12, who also carried a $500 \mathrm{~kb}$ duplication at $5 \mathrm{q} 23.3$ inherited from his clinically normal father; this was thought to be a benign variant-arr[hg19] 5q23.3(127,751,662-128,250,700)x3 pat.

All patients were found to share many of the clinical features previously reported to be characteristic of the distal 22q11.2 microdeletion syndrome, including global DD, ID, and mild 


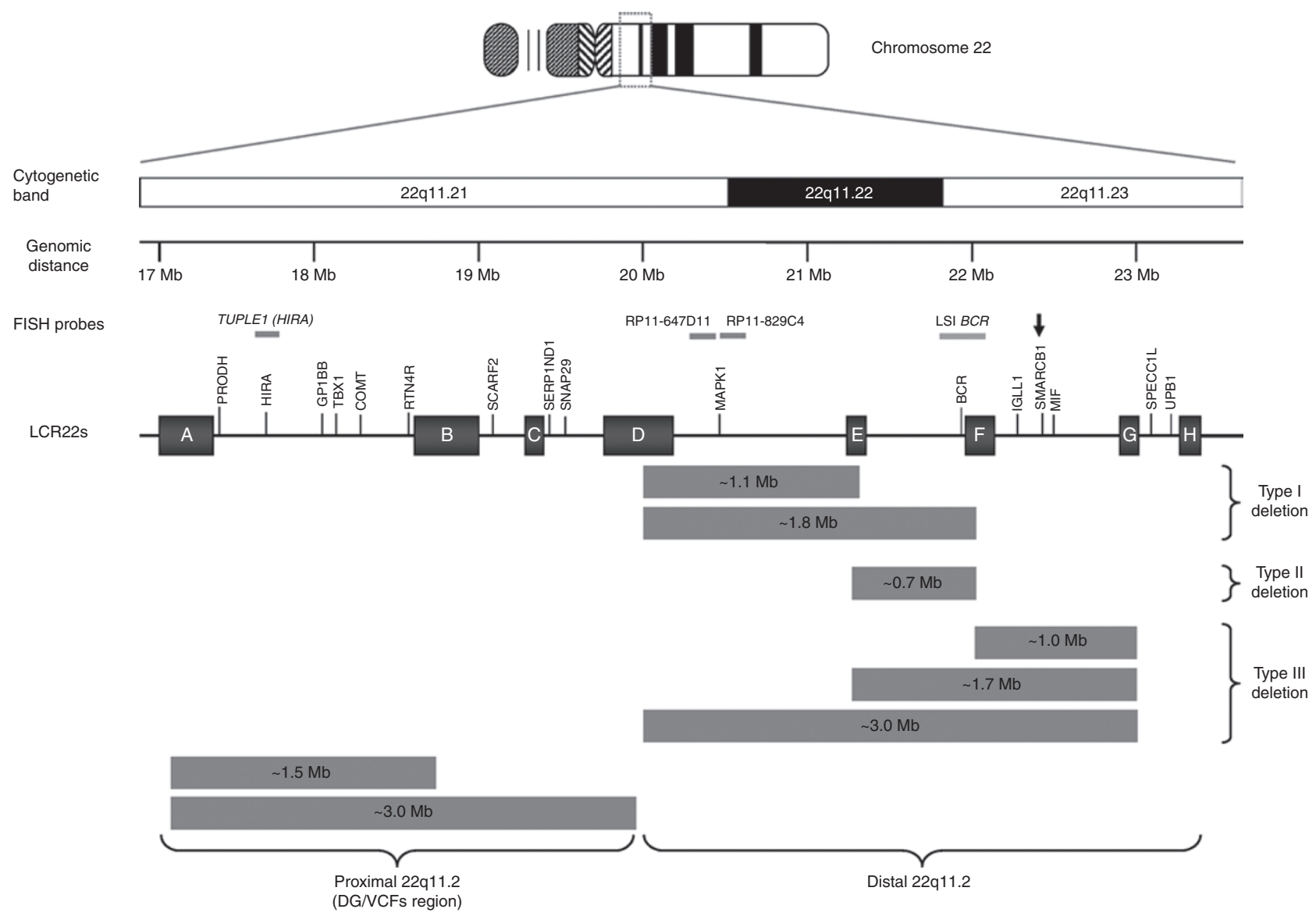

Figure 2 Schematic representation of the 22q11.2 region and the recurrent microdeletions reported in this region. Cytogenetic bands and genomic ruler are shown (hg18). FISH probes used are illustrated by the horizontal bars. The eight LCR22 clusters are illustrated by the green boxes and labeled A to H. The red horizontal bars below the map depict the reported deletions at both the proximal and distal 22q11.2 regions (refs. 7-18). The proximal 22q11.2 region corresponds to the DGNCFs typically deleted region. The distal 22q11.2 microdeletions can be categorized into three types: type I: with deletions flanked by LCR22-D and either -E or -F, type II: with deletions flanked by LCR22-E and -F, and type III: with any deletion in this region minimally spanning the LCR22-F to -G interval and encompassing the SMARCB1 gene (black arrow). DGNCFs, DiGeorge/velocardiofacial syndrome; FISH, fluorescence in situ hybridization.

dysmorphic features. However, there was a noticeable degree of phenotypic variability that could be partially explained by the genotypic variability. For all patients for whom we have gestational age data (12 of 13 patients), 9 patients were born prematurely with a gestational age ranging between 27 and 36 weeks. Those born prematurely were mostly delivered by C-section due to causes that included preeclampsia and oligohydramnios. Preterm birth was noted in eight of eight patients (100\%) with either the LCR22-D to -E deletion or the LCR22-D to -F deletion, whereas it was noted in one of four patients (25\%) with the small LCR22-E to -F deletion.

Prenatal growth restriction as evidenced by a low birth weight for gestational age $(<10$ th percentile) was noted in five patients, whereas seven patients had a birth weight between the 10th and 90th percentile. The birth weight was not available for patient 3. Five patients had a history of postnatal growth restriction, with height consistently below the 10th percentile, whereas six patients had postnatal growth that was within the normal range. In the two remaining patients, postnatal weight and height data were not available. Collectively, pre- and/or postnatal growth restriction was noted in seven of nine patients (78\%) with either the LCR22-D to -E deletion or the LCR22-D to -F deletion, whereas no growth restriction was noted in the four patients with the small LCR22-E to -F deletion.

Global DD and mild to moderate ID were universal clinical features in all patients. The DD was noticeably more prominent in language (Table 1). Eight patients suffered from various behavioral problems, which included social immaturity, talking to oneself, poor impulse control, anger issues, sleep problems, attention-deficit hyperactivity disorder, and autism spectrum disorders. Seven patients showed evidence of neurological problems, which included poor coordination, hypotonia, and seizures, with the most severely affected patient presenting with static encephalopathy. The behavioral and neurological problems were noted in all deletion types (Table 1).

Cardiovascular defects were noted in five of nine patients (56\%) with either the LCR22-D to -E deletion or the LCR22-D to $-\mathrm{F}$ deletion, whereas no cardiovascular defects were noted in the four patients with the small LCR22-E to -F deletion. The cardiovascular defects observed in our patients varied in 
severity and included patent ductus arteriosus, atrial and ventricular septal defects, and bicuspid aortic valve (Table 1).

Physical examination revealed nonspecific facial dysmorphic features in eight patients (Table 1). These included narrow long face or small triangular face, prominent forehead, bitemporal narrowing, flattened occiput, arched eyebrows, synophrys, narrow palpebral fissures, small upslanting eyes, deep-set eyes, low-set small ears, broad nasal root, short upturned nose, and pointed chin. Two patients had cleft lip and palate.

Minor skeletal abnormalities were noted in seven patients, including bilateral fifth-finger clinodactyly and valgus deformity in patient 1 , right claw hand with fused fourth and fifth fingers in patient 2 , third-finger clinodactyly with marked angulation of the left knee and second- and third-toe syndactyly in patient 3 , joint contractures in patient 6 , long tapered fingers in patient 8 , bilateral fifth-finger brachydactyly in patient 10 , and joint hypermobility in patient 12 . Other clinical problems noted in our patients included panhypopituitarism with septooptic dysplasia in patient 2 , history of pituitary tumor in patient 3 , ptosis in patient 6 , and truncal obesity with gynecomastia in patient 8 . Three patients (patients 2, 3, and 8) had small testes and/or small penis, with one of them also presenting with delayed puberty. Patient 9 also presented with retinopathy of prematurity, which progressed to retinal detachment and eventually she became blind in both eyes. She also suffered from left-ear hearing loss with narrow ear canals, as well as megacolon; she was G-tube-dependent for feeding.

\section{DISCUSSION}

The previously purported distal 22q11.2 microdeletion syndrome (OMIM 611867) in the region immediately distal to the DG/VCFs recurrent microdeletion region has been reported to be associated with a variable phenotype that includes core features in the form of preterm birth, pre- and/or postnatal growth restriction, DD, ID, behavioral problems, cardiovascular defects, skeletal abnormalities, and mild dysmorphic facial features. ${ }^{7-18}$ These deletions are mediated by nonallelic homologous recombination between the five telomeric LCR22s (LCR22-D to -H) in the distal portion of the 22q11.2 region (Figure 2). ${ }^{10}$ Distal 22q11.2 microdeletions that span the LCR22-F to -G interval encompassing the tumor suppressor SMARCB1 gene (also called INI1) have been reported to manifest many of the presenting features mentioned above but also have high incidence of malignant rhabdoid tumors in infancy and early childhood, predominantly in the kidneys and central nervous system, which necessitates tumor surveillance in these patients. ${ }^{19-24}$

Depending on the mediating LCR22s, the distal 22q11.2 microdeletions can vary in size between $\sim 700 \mathrm{~kb}$ and $\sim 3.0 \mathrm{Mb}$. To date, the distal 22q11.2 microdeletions have been grouped together as a single clinical entity despite the fact that these deletions comprise several partially overlapping and nonoverlapping deletions of varying size. In this study we present 13 new unrelated patients with variable-sized deletions in the distal 22q11.2 region. A near-universal feature noted in all except three of our patients (patients 11,12, and 13) is preterm birth with a gestational age that varied between 27 and 36 weeks. Patients 11,12, and 13 were born at full term and carried the small LCR22-E to -F deletion. Taken together, our results strengthen the association between the distal 22q11.2 deletions that span the LCR22-D to -E interval and preterm birth but suggest that preterm birth is not a common feature in patients with the small LCR22-E to -F deletion. Preterm birth is not a common feature of chromosomal aberrations in general, and has not been described in the DG/VCFs. It is worth noting that some of the severe presenting features observed in our patients can be directly attributed to very early preterm birth, as in the case of patient 9, who suffered from static encephalopathy and retinopathy of prematurity that was complicated by retinal detachment and blindness.

Approximately $78 \%$ (7 of 9 ) of the patients in this study with the 1.1-Mb LCR22-D to -E deletion and the larger 1.8-Mb LCR22-D to -F deletion, and whose birth weight and postnatal weight/height data were available, showed evidence of pre- and/ or postnatal growth restriction. The four patients with the small 700-kb LCR22-E to -F deletion had normal pre- and/or postnatal growth curves, which suggest that the critical region for growth restriction maps to the LCR-D to -E interval. This region encompasses 22 annotated RefSeq genes, including the MAPK1 gene. MAPK1 encodes mitogen-activated protein kinase 1 and has been proposed to be a candidate for the prematurity and/ or low birth weight in distal 22q11.2 microdeletions due to the fact that it is involved in placental development. ${ }^{12}$ These data suggest that pre- and postnatal growth restriction with short stature are common features of the distal 22q11.2 deletions that span the LCR22-D to -E interval but not of those that span the LCR22-E to -F interval.

Global DD and ID with a significant language delay component were noted in all our patients, including those with the small 700-kb LCR22-E to -F deletion. DD and/or ID have been reported in more than $80 \%$ of distal 22q11.2 microdeletion patients described in the literature to date and tend to be relatively mild to moderate in severity. ${ }^{7-18}$ Taken together, global DD and ID seem to be key components of the distal 22q11.2 microdeletions phenotype irrespective of the size and position of the deletion. Behavioral and neurological problems were also quite common in our patients in all deletion types. The behavioral problems included social immaturity, poor impulse control, anger issues, sleep problems, attention-deficit hyperactivity disorder, and autism spectrum disorders, whereas the neurological problems included poor coordination, hypotonia, and seizures, with the most severely affected patient presenting with static encephalopathy.

Congenital cardiovascular defects were observed in $~ 50 \%$ of distal 22q11.2 microdeletion patients and include septal defects, truncus arteriosus, bicuspid aortic valve, and cardiac dextrorotation. ${ }^{8,9,12,15,17,18}$ A similar frequency of cardiac defects was noted in our study, with five patients $(\sim 42 \%)$ presenting with a history of a cardiac defect, including patent ductus arteriosus, atrial and ventricular septal defects, and bicuspid aortic 
valve. It is worth noting that the four patients with the small 700-kb LCR22-E to -F deletion did not have history of a cardiac defect, again suggesting that the critical region for cardiac defects maps to the LCR-D to -E interval.

Only one patient with the small LCR22-E to -F deletion was previously reported in the literature. ${ }^{9}$ This patient had a congenital heart defect with normal growth, appearance, and psychomotor development. On careful examination, she was found to have slightly broad folding of the right helix and widely spaced inverted nipples. Her father carried the same deletion and had narrow palpebral fissures and low-set ears but no heart defects. Seven more patients were reported in the DECIPHER Database with this small LCR22-E to -F -deletion. ${ }^{25}$ The clinical features of five of them (patients 1579, 255108, 258704, 265081, and 271344) were available, and included mostly DD, ID, and mild dysmorphic features. No preterm birth, growth restriction, or heart defects were reported. This suggests that this small LCR22-E to -F deletion is relatively rare and/or has a mild phenotype. However, ascertainment bias due to the milder phenotype cannot be excluded, which might be a confounder in establishing the phenotypic correlations and relative frequency of this small deletion.

Dysmorphic facial features commonly reported in distal 22q11.2 microdeletion patients include arched eyebrows, deepset eyes, smooth philtrum, thin upper lip, hypoplastic alae nasi, and small pointed chin. ${ }^{7-18}$ Moreover, some patients present with cleft lip and/or cleft palate. Although some of our patients manifested some of these features, the majority of them presented with nonspecific, nondiagnostic features that were quite variable, as shown in the Results section.

In conclusion, we suggest that the recurrent distal 22q11.2 microdeletions do not represent a single clinical entity but vary depending on the specific mediating LCR22s and the intervening gene content. Our data suggest that the 1.1-Mb LCR22-D to -E deletion and the 1.8-Mb LCR22-D to -F deletion have common presenting features similar to the ones reported in the literature. The small 700-kb LCR22-E to -F deletion, however, seems to be relatively less frequent and to have a milder phenotype that is less likely to include preterm birth, pre- and postnatal growth restriction, and cardiovascular defects. Patients who carry this small deletion share with the larger 1.1-Mb and 1.8$\mathrm{Mb}$ deletion patients the other common presenting features, including the DD, ID, and minor nondiagnostic dysmorphic features. This observation, however, waits to be confirmed in other studies due to the relatively small number of patients with the small 700-kb LCR22-E to -F deletion presented here and in the literature. On the other hand, deletions in the distal 22q11.2 region that span the LCR22-F to -G interval including the SMARCB1 gene have been reported in multiple studies and were shown to be also variable in size and share many of the presenting features of the distal 22q11.2 microdeletion phenotype discussed earlier, in addition to a very high incidence of malignant rhabdoid tumors in infancy and early childhood. ${ }^{19-24}$

Accordingly, we propose categorizing the distal 22q11.2 microdeletions into three genomic types: type I: with deletions flanked by LCR22-D and either -E or -F; type II: with deletions flanked by LCR22-E and -F; and type III: with any deletion in this region minimally spanning the LCR22-F to -G interval and encompassing the SMARCB1 gene (Figure 2). All three deletion types are thought to be pathogenic and are most often de novo. They all share many of the presenting features mentioned above but also have their unique features and risks. Type II deletion seems to be relatively less frequent and to have a milder phenotype that is less likely to include preterm birth, pre- and postnatal growth restriction, and cardiovascular defects, whereas type III deletions are characterized by a very high incidence of malignant rhabdoid tumors in infancy and early childhood. This underscores the need for accurate breakpoint mapping and sizing of these deletions in clinical practice.

\section{ACKNOWLEDGMENTS}

We are extremely grateful to the patients and their families for their participation and cooperation in this study. DECIPHER acknowledgment: This study makes use of data generated by the DECIPHER Consortium. A full list of centers that contributed to the generation of the data is available from http://decipher.sanger. ac.uk and via e-mail from decipher@sanger.ac.uk. Funding for the project was provided by the Wellcome Trust.

\section{DISCLOSURE}

The authors declared no conflict of interest.

\section{REFERENCES}

1. Shaw-Smith C, Redon R, Rickman L, et al. Microarray based comparative genomic hybridisation (array-CGH) detects submicroscopic chromosomal deletions and duplications in patients with learning disability/mental retardation and dysmorphic features. J Med Genet 2004;41:241-248.

2. Girirajan S, Brkanac Z, Coe BP, et al. Relative burden of large CNVs on a range of neurodevelopmental phenotypes. PLoS Genet 2011;7:e1002334.

3. Cooper GM, Coe BP, Girirajan S, et al. A copy number variation morbidity map of developmental delay. Nat Genet 2011;43:838-846.

4. Hastings PJ, Lupski JR, Rosenberg SM, Ira G. Mechanisms of change in gene copy number. Nat Rev Genet 2009;10:551-564.

5. Carlson C, Sirotkin H, Pandita R, et al. Molecular definition of 22q11 deletions in 151 velo-cardio-facial syndrome patients. Am J Hum Genet 1997;61: 620-629.

6. Scambler PJ. The 22q11 deletion syndromes. Hum Mol Genet 2000;9:24212426.

7. Rauch A, Pfeiffer RA, Leipold G, Singer H, Tigges M, Hofbeck M. A novel 22q11.2 microdeletion in DiGeorge syndrome. Am J Hum Genet 1999;64:659666.

8. Saitta SC, McGrath JM, Mensch H, Shaikh TH, Zackai EH, Emanuel BS. A 22q11.2 deletion that excludes UFD1L and CDC45L in a patient with conotruncal and craniofacial defects. Am J Hum Genet 1999;65:562-566.

9. Rauch A, Zink S, Zweier C, et al. Systematic assessment of atypical deletions reveals genotype-phenotype correlation in 22q11.2. J Med Genet 2005;42:871-876.

10. Shaikh TH, O'Connor RJ, Pierpont ME, et al. Low copy repeats mediate distal chromosome 22q11.2 deletions: sequence analysis predicts breakpoint mechanisms. Genome Res 2007;17:482-491.

11. Mikhail FM, Descartes M, Piotrowski A, et al. A previously unrecognized microdeletion syndrome on chromosome 22 band q11.2 encompassing the BCR gene. Am J Med Genet A 2007;143A:2178-2184.

12. Ben-Shachar S, Ou Z, Shaw CA, et al. 22q11.2 distal deletion: a recurrent genomic disorder distinct from DiGeorge syndrome and velocardiofacial syndrome. Am J Hum Genet 2008;82:214-221.

13. Rødningen OK, Prescott T, Eriksson AS, Røsby O. 1.4Mb recurrent $22 q 11.2$ distal deletion syndrome, two new cases expand the phenotype. Eur J Med Genet 2008;51:646-650. 


\section{ORIGINAL RESEARCH ARTICLE}

14. Bruce S, Hannula-Jouppi K, Puoskari M, et al. Submicroscopic genomic alterations in Silver-Russell syndrome and Silver-Russell-like patients. J Med Genet 2010;47:816-822.

15. Madan S, Madan-Khetarpal S, Park SC, et al. Left ventricular non-compaction on MRI in a patient with 22q11.2 distal deletion. Am J Med Genet A 2010;152A:1295-1299.

16. Verhoeven W, Egger J, Brunner H, de Leeuw N. A patient with a de novo distal 22q11.2 microdeletion and anxiety disorder. Am J Med Genet A 2011:155A:392-397.

17. Tan TY, Collins A, James PA, et al. Phenotypic variability of distal 22q11.2 copy number abnormalities. Am J Med Genet A 2011;155A $1623-1633$.

18. Fagerberg CR, Graakjaer J, Heinl UD, et al. Heart defects and othe features of the 22q11 distal deletion syndrome. Eur J Med Genet 2013;56: 98-107.

19. Jackson EM, Shaikh TH, Gururangan S, et al. High-density single nucleotide polymorphism array analysis in patients with germline deletions of 22q11.2 and malignant rhabdoid tumor. Hum Genet 2007;122:117-127.
MIKHAlL et al | A categorization system for the recurrent distal 22q11.2 microdeletions

20. Lafay-Cousin L, Payne E, Strother D, Chernos J, Chan M, Bernier FP. Goldenha phenotype in a child with distal 22q11.2 deletion and intracranial atypical teratoid rhabdoid tumor. Am J Med Genet A 2009;149A:2855-2859.

21. Eaton KW, Tooke LS, Wainwright LM, Judkins AR, Biegel JA. Spectrum of SMARCB1/INI1 mutations in familial and sporadic rhabdoid tumors. Pediatr Blood Cancer 2011;56:7-15.

22. Beddow RA, Smith M, Kidd A, Corbett R, Hunter AG. Diagnosis of distal 22q11.2 deletion syndrome in a patient with a teratoid/rhabdoid tumour. Eur $J$ Med Genet 2011:54:295-298.

23. Toth $\mathrm{G}$, Zraly CB, Thomson TL, et al. Congenital anomalies and rhabdoid tumor associated with 22q11 germline deletion and somatic inactivation of the SMARCB1 tumor suppressor. Genes Chromosomes Cancer 2011;50:379-388.

24. Chakrapani AL, White CR, Korcheva V, et al. Congenital extrarenal malignant rhabdoid tumor in an infant with distal 22q11.2 deletion syndrome: the importance of SMARCB1. Am J Dermatopatho/ 2012;34:e77-e80.

25. Firth HV, Richards SM, Bevan AP, et al. DECIPHER: Database of Chromosomal Imbalance and Phenotype in Humans Using Ensembl Resources. Am J Hum Genet 2009;84:524-533. 